\title{
Vie pastorale et industrie dans le Val d'Ariège (canton des
}

\section{Cabannes)}

\section{Georges Bertrand}

\section{Résumé}

La vie pastorale a survécu à la décadence du système agro-pastoral en devenant une ressource d'appoint pour beaucoup d'ouvriers (usine des Talcs) et de retraités. Elle a conservé sa structure traditionnelle (estivage communautaire, production d'animaux jeunes) et, à part quelques améliorations (double agnelage, moto-faucheuses), elle ne paraît pas susceptible de modernisation (manque d'esprit coopératif, pas de place pour les prairies artificielles). Elle représente cependant $95 \%$ du revenu des montagnards purs, 10 à $40 \%$ de celui des ouvriers- éleveurs, 15 à $70 \%$ de celui des retraités-éleveurs. Elle est un frein à la prolétarisation. Mais l'effondrement démographique compromet son avenir.

\section{Citer ce document / Cite this document :}

Bertrand Georges. Vie pastorale et industrie dans le Val d'Ariège (canton des Cabannes). In: Revue géographique des Pyrénées et du Sud-Ouest, tome 32, fascicule 1, 1961. pp. 63-73;

doi : https://doi.org/10.3406/rgpso.1961.4525

https://www.persee.fr/doc/rgpso_0035-3221_1961_num_32_1_4525

Fichier pdf généré le 19/07/2018 


\section{VIE PASTORALE \\ ET INDUSTRIE DANS LE VAL D'ARIËGE \\ (Canton des Cabannes) \\ par Gegrges BERTRAND}

Les 25 eommunes du anton des cabannes (3 700 habitants en 1954) forment un ensemblo homogene. Axé sur le « Val d'Ariège », le canton est termé, à l'amont, par les rerrous glaciaires

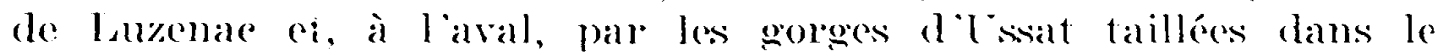
caleatre urgonien. Il déborde al: Nond sur le massit du l'abe ot au Sud sur les hautes surfaces de l'Aston, conglohant ainsi, entre i $000 \mathrm{~m}$ et $2570 \mathrm{~m}$ daltitude, de vastes pâtumages qui lui dombent une incontestable reation pastorale (fig. 1 ).

la vie pastorale sest maintenue, malgré la décoldence de lam-

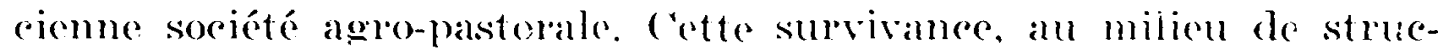
tures économiques et sociales nouvilles, pose plusieurs problemes. Dans quelle mesure la vie pastorale résiste-t-elle on s'arlapte-t-elle au système économique aetuel et, surtout, quel est son rôle dans la nouvelle société montagnarde (1)!

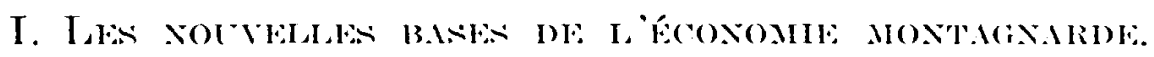

On assiste a un bouleversement des structures économiques ot soeiales. les ressomeres traditiomnelles décelinent ot d autres, à caraletere extra-montagnard, les remplacent rapidement.

1. La base vivrière se rétrécit de plus en plus. - La terroir agricole, eoneentré au fond des vallées of sur les basses pentes des

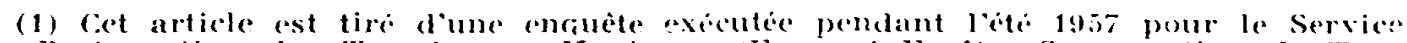
de Restaturation des Terrains en Montagene (Faux et Forèts, Conservation de Toulbuse) at qui nous adressons nos vifs remereiements. (ce travail a ite mis à jour en juin 19tio, à loceasion du $\mathrm{XVl}^{*}$ Congres de la Federation des Societes academiques

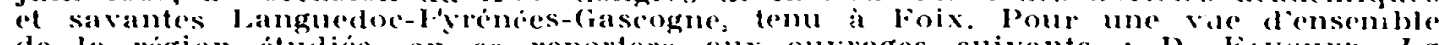

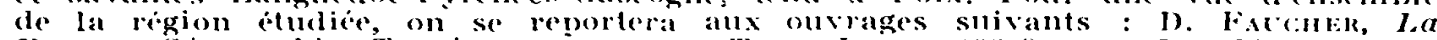

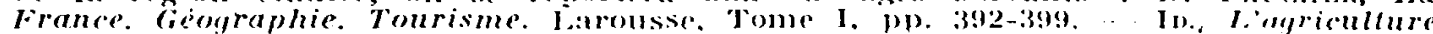

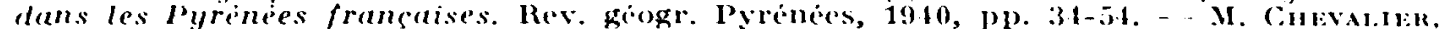

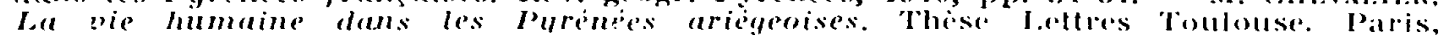

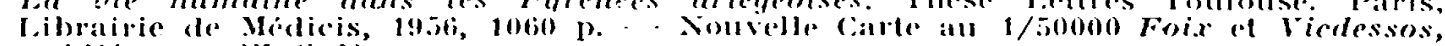
publice par i'I.
} 
somlanes, no comprend que 2000 heretares, soit à peine $6,5 \%$ de la superficie totale. Les labours noceupent que $43 \%$ du domaine rultivé. La reste est comsaréé aux prairies de fauche.

Il ny a done pas un demi-hectare labourable par habitant (4) ares). Sur la haute soulane du Tabe. le territoire communal de Caychax (40 habitants, 9 familles) se répartit de la manière suivante (2) :

$\begin{array}{lrlr}\text { terrains incultes } & 350 \text { ha } & \text { prés naturels } & 23 \text { ha } \\ \text { bois } & 153 \text { ha } & \text { divers } & 5 \text { ha } \\ \text { terres labourables } 27 \text { ha } & \text { total } & 562 \text { ha }\end{array}$

Les propriétés - l'exploitation directe est de règle - n'ont en moyemne que 3 à 5 ha et la plus étendue ne dépasse pas 15 ha. Hlles sont très moreclécs. La superficie des pareelles est de lordre de 10 à 35 ares. C'est ainsi qu une propriété de 10 ha est divisée en plus de 30 parreelles. ('ot émiettement. rendu nécessaire par la vigueur des pentes, s'est alcompagné d'édification de terrasses, alljourd hui roulantes, entre lesquelles les rharrettes ne peuvent pas circuler. Ia mécanisation est limitée à l'omploi de quelques motolaucheuses. Les tracteurs ne sont. utilisés que dans les champs plats de la rivière ou sur les nhaces forestiers pour le débardage des grumes.

les montagnards sont restés fideles au vieux principe dautoconsommation. Ia culture des pemmes de tere lemporte aree 129 ha. cest encore, aree la viande de pore. la hase de lalimentation. le blé vient ensuite (105) ha) maintenu par routine. (a) les rendements sont dérisoires. Il ne eouve pas les besoins de la consommation locale. Ie mais-grain, qui narrive pas toujours a maturité, orenpe 5o ha et les céréales secondaires 75 ha (méteil, aroine de printemps, blé noir). Il y a enfin tis ha de vignes à piquette dispersées sur les pentes caillouteuses de soulanes calcaires (Albies, Vobre, Verdun, ete.). Ises cultures sont insuffisantes et beaucoup de montagnards qui travaillent à l'usine ne les considèrent que comme un appoint.

2. L'emprise industrielle est un fait majeur. - Ia tradition industrielle à toujours été forte dans la vallée de l'Ariège, riche en minerai de fer et en main-d'ourre. Te canton des Cabamnes possédait les mines de fer de Lassur et les forges à la catalane de Gudanes près d'Aston, Cependant, jamais l'industrie n'a, autant qu'aujourd'hui, coneurrence le genre de vie agro-pastoral. Il y a un demi-siecle, les actirités industrielles s̈inséraient sourent dans le calendrier aquieole

(2) Recensoment agricole 19:66. 
dont elles oceupaient les temps morts. Elles représentaient un complément alors quielles sont devonues la ressouree essentielle.

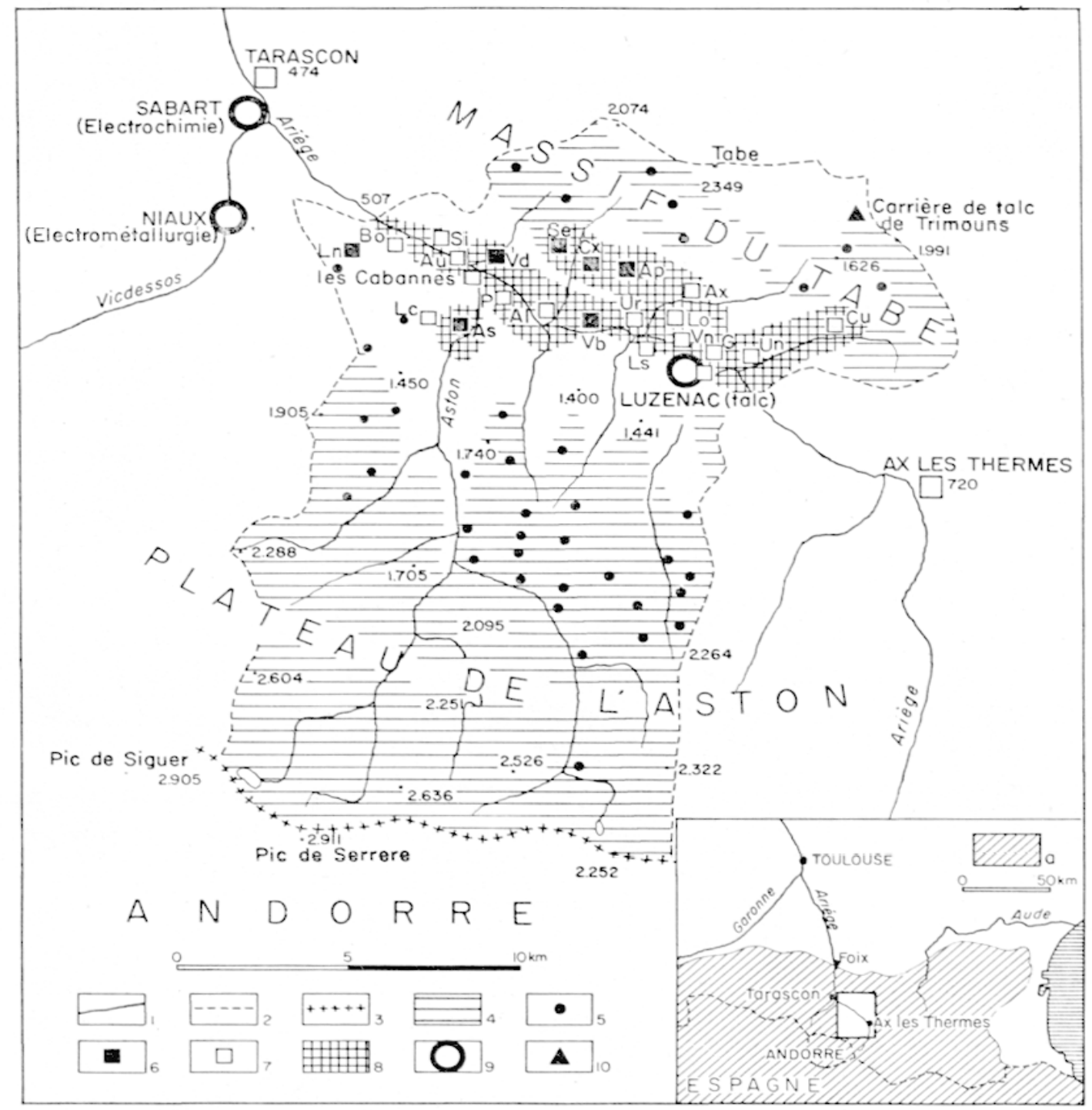

FIG. 1

La vie économique du canton des Cabannes.

1. Torrents. -. 2. Limite du canton. -. 3. Frontiere. 4. Estive. -.. 5. Jasse. - 6. Village à prédominance asro-pastorale. 7. Village d'ou-

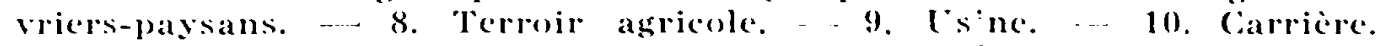
Al. Albies. - Ap. Appy. - As. Aston. - Au. Aulos. - Ax. Axiat. Be. Bestiac. - Bo. Bouan. Cu. Caussou. -.. C.x. Caychax. - G. Garanull. I.e. Larcat. -. I,n. Iarnat. - Is. Lassur. - Io. Lordat. - P. Pech. .. Se. Senconac. - Si. Sinsat. In. Inac. - I'r. I'rs. - Vh. Vebre. Vd. Verdun. - Vn. Vernaux.

Files orempent 600 persommes domiciliées dans le eamton, soit $15,9 \%$ de la population totale. On compte trois ecotres d'embauche: 
lusine électro-métalhurgique de Niaux dans la vallée voisine du Viedessos, lusine électro-chimique de Sabart à côté de Tarascon ef surtout la Société des Tales de Luzenac. Sur un total de 1020 ouviers, cette dernière occupe $\$ 13$ hommes du canton (3). Parmi ces derniers, 169 travaillent à la carrière des tales de Trimouns, à $1400 \mathrm{~m}$ d altitude. Pour des raisons climatiques (persistance du manteau neigeux) la carrière ne fonctionne que $d u 1^{\text {er }}$ avril au $1^{\text {er }}$ novembre. Ises ourriers y sont nourris et logés en dortoir. I'usine, située entre Luzenac et Garanou, occupe 234 ouvriers du canton.

Le travail est pénible et malsain. Il y a de nombreux cas de silicose parmi les ouvriers de la Société des Tales, et d'intoxication par les produits ehimiques dans les autres usines. Les salaires de base restent relativement bas et les primes de rendement poussent au surmenage. Le travail s'effectue par postes de huit heures, ce qui permet aux ouvriers davoir d'autres occupations, en dehors de ceux qui travaillent à la. carrière.

2. Les retraites sont un complément substantiel - I'embauche industrielle est on effet insuffisante pour retenir tous les jeunes gens et elle ne concerne pas du tout les jeunes filles. Ises jeumes quittent la montagne pour aller travailler dans le Bassin d'Aquitain ou dans les grandes villes : Toulouse, Paris, Maseille. Beaucoup y reviennent au moment de leur retraite. ('est un par's de migrants-viagers. Actuellement, les retraités représentent 9,8\% de la population totale. Il y a ainsi de véritables villages-refuges : $\Lambda$ ppy, 20,4 \%; Lassur, $17,6 \%$; Sinsat, $15 \%$, ete.

Dans cette vallée aux revenus médiocres, on ne doit pas sousestimer le rôle économique de la retraite. C'est une source appréciable d'argent frais; d'autro part, beaucoup de retraités s'occupent d'une exploitation ou d'un troupeau. Ces activités ne sont pas totalement. dépourvues d'arenir puisqu'on assiste à un renouvellement assez rapide des retraités. Mais il semble que, depuis deux ou trois ans, les retours all pays natal soient moins fréquents; les retraités qui s'installent dans le canton paraissant délaisser les activités agro-pastorales.

\section{LE MLANTIEN DI SYSTÈME PASTORAI TRADITIONNEL}

La vie pastorale était la clé de roûte de l'ancienne économic. Aujourd'hui, elle a perdu beaucoup de son importanee mais a conservé son organisation traditionnclle. Ise sistème pastoral repose sur

(3) Ia Socicte des Tales engage beatcoup détrangers : Portugais, Italiens ou surtout lispagnols. 
l utilisation alternée de deux domaines eomplémentaires mais dissociés dans lispace : les prairies de fauche de la rallée et, 1000 à 1 500 m an-dessus, les hauts pâturages ou estives que lion atteint par de médiocres sentiors.

1. La décadence de l'estivage. - Ia dépécoration est un tait general. Les 20000 hectares d estives constituent une grande richesse naturelle. Les éleveur:s du canton n y envoient que 2600 à 2800 bovins of $u n$ beu plus de 7000 ovins (4). Il fant $x$ jomelre quelques animaux du Viedessos, de la région de Tarascon et de Montferrier. Au total, les cliecelits ne dépassent pas 3000 bovins et 10000 ovins, soit une vache pour 7 hectares de pelouse et un mouton pour 2 heetares. Au début du xix" siècle. l'Aston nourrissait à lui seul plus de 30000 ovins. Ial sous-exploitation entraîne la dégradation des pelouses : les jasses ne sont phus fumées par les troupcaux (5), les chemins et les cabanes pastorales sont mal entretenus. Comme toute la haute Arioge, le canton des Cabannes est un pays naisseur et producteur de viande. Ia vie pastorale repose sur deux rigoureuses races it viande : la race bovine gaseome et la race ovine tarascomnaise. ('" sont des animaux rustiques, aptes à supporter pendant einq mois le sćjour sur les estives ot à rester, pendant les sept autres mois, sols-alimentés ot onfermés presclue tout le temps dans des étables nauséabondess.

La:; deux produits essentiels sont le brau (veau mâle) de 7 mois pesant de 250 a $300 \mathrm{~kg}$ et läneau de printemps. ('et élevage d animalux jeunes est la seule spéeulation conciliable avec la diffieulté d acees anx estives. On ne voit pas comment ou pourrat eréer un systemo de rruitières de type jurassien (on l'a tenté vers 1903) ou de lateries de lype Ropuefort, diatant plus qu'on se trouve en présence d iun sistème pastoral de glande montagne (6).

la vie pastorale repese, en effet, sur l itilisation communautaire des estives. ('haqua printemps, rels le 20 mai, on rassemble les animaux du village ou, de plus en plus à eause de la faiblesse des offectits, de plusiens villalges (syondicat pastoral Lrs-Vêbe-hassur par cxemple). On eonstitue 10 eachories de 90 à 230 bêtes adultes ef 11 troupealux d ovins. ('haque troupeau est confié à un seul vacher ou a un seul berger. Après un bref séjour dans les prairies de fauche, les animaux erimpent directement sur les estives, où les parcours

(1) Chitres domnes par l'enquete directe.

(i) Ja jasse est un scetenr do paturage abrite, situe près d'une abane, ou lon reunit chature soir le trompratu.

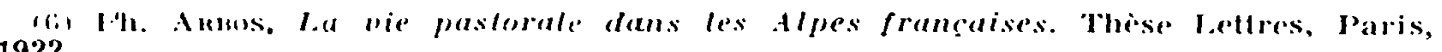
1922 . 
sont minutieusement réglés par les droits d'usage. Ils circulent de jasse en jasse jusque rers la mi-octolore date du retour dans la vallée. les ovins, plus sensibles anx intemperies, montent plus tand et redeseendent plus tôt. I, équipement des estives est resté très rudimentaire. Soil $y$ a asso de cabanes, dont rertaines sont presque confortables, les alneruvoirs of surtout les bons chemins pastoraux manquent. On nete epondant quelques progres. ("est almsi que de syndicat tro-lobre-Lassur a fait ronstruire une route pastorale aceessible aux jecps, qui débloque les estives de l'Aston rentral. Elle

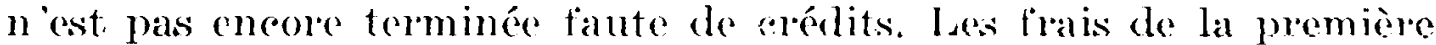
tranche de travaux ont été couverts par la vonte de coupes de bois. La vie des berogers reste néammoins très rude. Le recrutement français est presque tari et lon fait appel à des bereers aragonais on andorrans. Le bétail, isolé, ne recoit pratiquement aucun soin : de

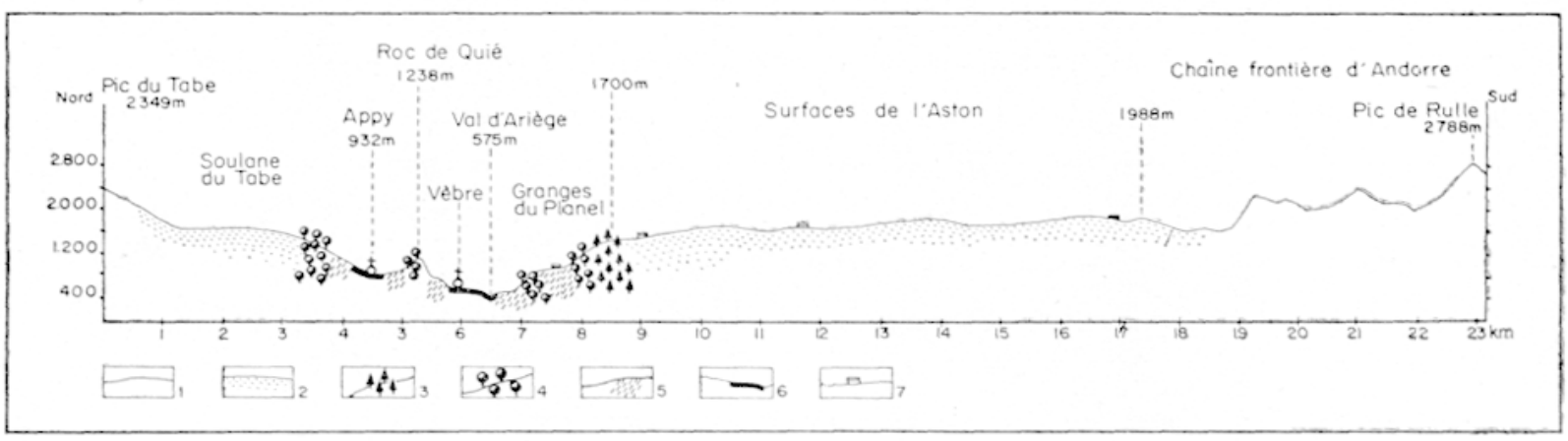

Fig. 2

L'étagement des ressources dans le Val d'Ariège.

1. Rochers, - 2. Pelouses alpines (estives). - 3. Forêt de résineux. ... t. Forêt de feuilus. - 5. Prairies de fauche. 6. Cultures. - 7. Granges et cabanes de bergers.

lourdes pertes sont enregistrées parmi les jeunes (maladie, acoidents) et au moment du vêlage.

Liorganisation de l'estivage, si impartaite soit-elle, n'en constitue pas moins une lourde charge pour des rommunautés villageoises en pleine décadence démographique. Boaucoup de villages renoncent à constituer une vacherie. Sur la soulane du Tabe, on assiste à une évolution du sastime pastoral. I ses communes afferment une partie de la montagne à un entrepreneur de transhumance qui introduit des animaux étrangers of prend on charge oo qui reste du bétail autochtone. Laeffort isolé de quelquess jemes élevenrs, qui se laneent dans la matique du double agnelage annuel, semble condammé par labandon massif des autres membres de la collectivité. Le systeme pastoral, tel qu il est concull, ne survirra pas à la désagrénation des communautés villageoiscs. 
2. Les problèmes de l'hivernage et da la commercialisation. - Dans lommédiat. lim des problemes les phus aigus est eolui d: lhivernage. Le nombre de bêtes qui hivernent est fonetion de la

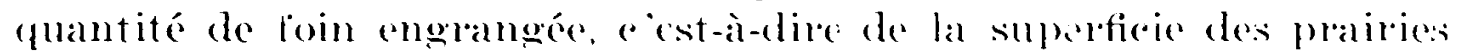
de tauche et, de plus en plus, de la maintel amve disposible au moment de la fenaison.

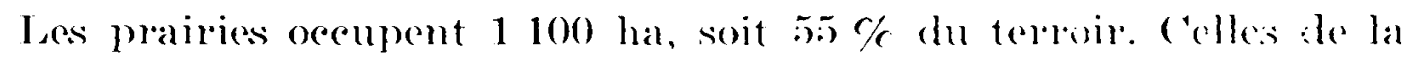
rivière domment un regain ot peurent être fanchés méaniquement. Les prés de versants ne doment quime eoupe. le travail is 'y effectue à la main et le transport à des l homme on de mulet; ils somt en voie d'abandon. Malgré de récents progress. les prairies artificiolless ne eourrent que 250 hectares. Jleme si toute la zone cultivere etait rouchee en prairies artifieiellew. on ne disposerait pas dassez de fourage pour lohier. Tal tamshumance inverse a été un palliatif à la pémurie de foin. Elle n intéresse plus que quelques troupeatux dovins de Vebre et de Ferelun qui hivernent dans les ('orbières: orientales.

Ce sont les foires de Tarascon et d'Ax qui compensent le désépuilibre saisomier. A l'automme, le montagnard rend tous les braus et les vaches réformées, ne conservant dans ses éables que les adultes et quelques volles pour la reproduction. Au printemps, il achète aux élevenrs de la plaine de jemes animaux qui, parfois. doublent l'effectif des troupeaux.

Ia commercialisation est le point le plus labhle de léeonomie pastorale. Chatgue eleveur rend pour son prople eompte. Ia phupart des montagnards se contentent dailleurs de fréguenter les foires d Ax et de Talasison. Ils ne sortent qu exceptionnellement du département. Iaes fluctuations des prix de ces dernieres ammées ont eté vivement ressenties, surtout à lanutomne 1959, où la marege dest bénéfices a été très taihle pour beancoup de petits éleveurs.

la vie pastorale est donc une simple surviance. Hille sest maintemue grâce à sa partaite adaptation aux eonditions maturelles. Ma is elle est de plus en plus désadaptée aux conditions de la production moderne. Il n'en reste pas moins qu chlle constitue, pour que? ques ammées encore, un élément non négligeable de léeonomie montasnarde.

III. T.A VIF P.ASTOR.ALE, RESOTRCE D APPOINT.

Grande originalité. en effet. du canten des ralhamnes. l'élevage est

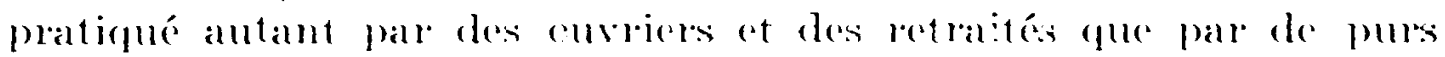
montagnards. ("est que rester forme délevage demande asse\% pou de travail. Is contretien du bétail, nul pendant la stabulation hirer- 
nale, ne pose de problemes de main-d epurre qu à lépoque de la fenaison. Aussi, rares sont les familles qui ne possedent pas quelques animaux. $52 \%$ des éleveurs ont moins de 4 hovins adultes, $43 \%$ en

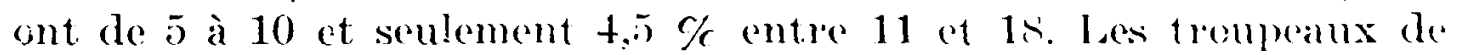
moutons comptent en moremne 90 à 110 têtes. Cependant, les jeunes de lamnée arrivent presque à doubler les cffectitis. L importance des troupeaux varie beaucoup suivant les catégories d'éleveurs.

1. Les types d'éleveurs et leurs revenus. - les élevils jill's, environ 20 à $25 \%$ de la population tetale, ont conservé mo qenre de vie agro-pastoral. Ce sont surtout des persomnes ágés. I)ans la commune d'Aston, la. moitié des élevenus ont plus de 60 ans et aucoun n a moins de 30 ans (7). (On y comptr:

3 éleveurs de 30 à 39 ans $\quad 14$ élevelues de 60 à 69 an:;

6

de 40 à 49 ans

j)

de 70 à 80 ans

10 de 50 à 59 allis soit 38 au total.

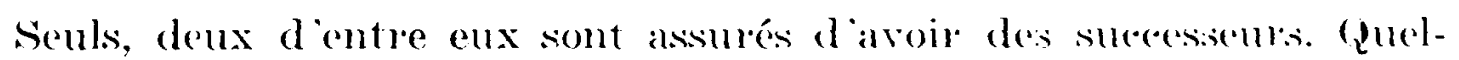
ques jeunes éleveurs, qui se sont trourés à la tête de weoses axploitations, sont restés à ba terre. Ils coustituent lélément druamique du canton. Ils siègent dans les municipalités et participent à lamélioration agricole (extension des prairies altificielles, achats de motofaucheuses). Mais ils ne sont que deux ou trois far commune.

La rente des animaux cist leur seuk souree d arent liquide. Sour; arons caleulé quelques revenus pastoraux pour l'amnée 1957. Tou; les chiffres s'entendent nets des frais dentretien du troupeau.

\begin{tabular}{|c|c|c|}
\hline COMMUNE & EFFECTIF DU TROUPEAU & $\begin{array}{c}\text { REVENU ANNUEL } \\
\text { en } 1957 \\
\text { (anciens francs) }\end{array}$ \\
\hline $\begin{array}{l}\text { 1. Aston } \\
\text { 2. Aston } \\
\text { 3. Vère } \\
\text { 4. Vère } \\
\text { 5. Axiat }\end{array}$ & $\begin{aligned} & 4 \text { vaches } 3 \text { veaux } \\
& 6 \text { vaches }-5 \text { veaux } \\
& 10 \text { vaches }-7 \text { veaux } \\
& 8 \text { vaches } 4 \text { veaux } \\
& \text { plus } 100 \text { moutons } \\
& 120 \text { brebis avec pratique } \\
& \text { du double agnelage }\end{aligned}$ & $\begin{array}{r}200000 \\
350000 \\
550000 \\
300000 \\
\text { Jlus } \quad 500000 \\
1000000\end{array}$ \\
\hline
\end{tabular}

Lälevage ovin rapporte done heancoup plus que celui des boxins.

(7) Il s'agit de lagge de lexploitant reel at non celuj du proprictaire legal fvicux parents par exemples. 
Mais il est délicat et demande davantage de temps. Les moutons étant plus fragiles que les vaches. l'estivage est écourté de plus d 'un mois, ce qui pase le problème de la garde du troupeau tamilial dans la vallée. Un membre de la famille doit s'y consacrer entièrement. C'est une véritable rocation. Aussi, lélevage ovin est-il lapanage des purs montagnards. Celui des bovins ne commence à être rentable quà partir d un trompeau de 5 à 6 vaches, cest-à-dire nettement au-dessus de la moyemne du canton (t vaches). Les petits éleveurs vont donc s'embaucher à l'usine, sans pour autant abandonner leur troupeau.

Les ouvriers-éleveurs (10-12\% de la population totale) forment une gamme sociale très nuancée qui va de l'ouvrier-chef d'exploitation, assumant la direction du troupeau, à l'ourrer qui aide sa famille à temps perdu, sans compter les nombreux ourriers qui ne passèdent pas eux-mêmes de bétail, mais qui se louent au moment de la fenaison.

En 195i, le salaire moren annuel de louvrier était de 400000 à 450000 anciens franes. Les troupeaux des ouvrepes-éleveurs comptaient de 2 à 5 bovins, soit 111 revemu net de 50000 à 200000 anciens flanes, ee qui represente de $10 \%$ à $40 \%$ du salaire. I asssociation de l’industrie et de lélevane est considérée comme une bome solution par les intéressés qui voient allgmenter leurs petites ressources, ot aussi par le patronat qui peut maintenir, au milieu du calme social, des salaires relativement has. Sur le plan humain, c'est une solution médiocre. Le travail double, mais pas le profit. Surtout, elle entraine ume désaffection progressive à l'éand de la vie rurale.

Les retraités-éleveurs (6 à $7 \%$ de la population totale) participent activement au maintien de la vie pastorale. Dans plusienrs villages, le tiers des ébeveurs sont des retraités (ecndarmes, wareons de caf'é, etc.). On distingue deux types de retraités-éleveurs. I es fonetionnaires retraités encore jeunes et assez aisés $(300000$ à 350000 an(ciens francs de retraite anmuelle), entretiennent de oros troupcaux qui augmentent leurs revenus dans la proportion de 15 à $25 \%$. I es retraités d un emploi civil qui ne hénéficient que de la retraite des vieux travailleurs salariés (une centaine de milliers de franes en 1957) trouvent $u$ appoint indispensable dans lólevage, qui peut accroitre leurs ressources des deux ticrs.

Ces catégories sociales ne sont pas nettement tranchées. Flles s'amalgament. au sein des mêmes familles et le décompte exact des différentes ressoureces est impossible à établir. Soit lexemple du village de caychax : il se compose de to habitants répartis an 9 familles; rhaque forer posiede une petite exploitation at un troulpeau de 3 à 10 vaches; un seul vit uniquement des revenus de la 
terre et du bétail; trois tirent lessentiel de leurs ressourees des salaires ourriers versés par l usine des Tales; quatre, formés de peisomnes âgées, subsistent grâce au soutien financier de leurs entants qui travaillent à la ville; le dernier bénéficie d une pension de guerre. Ial coupure sociale et psychologique la plus nette est celle qui opposio les non-éleveurs (ouvriers de luzenac et habitants du boure des Cabannes) aux éleveurs, qu ils soient montagnards purs, ourriers; ou retraités.

Ia vie pastorale apparait done comme un important élément de la vie sociale et de l'équilibre économique. A-tedle une influence sur l'érolution démographique (8) ?

\section{La vie pastcrale et le déclin des communautés villageoises.}

- Ises villages agro-pastoraux des hautes soulanes sont en pleine déadence. le vioillissement de la population y est frappant. A Caychax, par exemple, la répartition par groupes d'âge est la suivante (1954) :

$$
\begin{aligned}
& 0-9 \text { ans : } 2 \% \\
& 10-34 \text { ans : }: 27 \% \\
& \text { 35-60 ans: :50 \% } \\
& \text { plus de 6:5 ans : } 21 \% \\
& \text { moins de } 35 \text { ans : }: 29 \% \\
& \text { plus de } 35 \text { ans : } 71 \%
\end{aligned}
$$

A Larcat, entre 1950) et 1960, 17 décés ont été déclarés, matis alucune naissance : on va fermer l'école. le genre de vie agropastoral paraît définitivement condammé par l'évolution démographique. Pourtant, un troupeau de 6 à 7 raches équiraut à un salaire d'ourrier et une centaine d ovins rapporte bien davantage. De l'avis des intéressés, le travail de l'eurrier est plus pénible que celui de

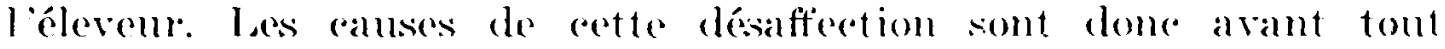
prychologiques. Cette mentalite est surtout sensible che\% les jemes; filles qui ne sombatent pas se marier aree mu clereur. Dans le vilaage d Aply (49 habitants) on compte $T$ célibataires masedins entio 20 et 40 ans. Il taut recomnatro que le travail de:s femmes est particulièrement pénible à la montagne. Il cest mêlae juée légralant par les intéressées.

la situation démographique des villages dourriers-palisans est légèrement meilleure, car l'émigration des jennes est fremée par les possibilités locales d embauche. ("est le cas d'Lnar (1s) habitants). où la preamide des âges est mieux équilibrée qua carohax :

\footnotetext{
(s) Nous ne reviendrons bas sur levolution demographique densemble. Voir:

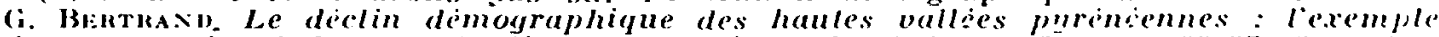
du canton des cabannes (Ariège). Rev, géogr. Pyrénés, 1959, ?p. \$2-87, 2 cartes.
} 


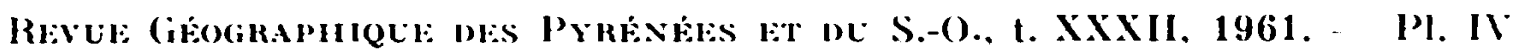

VIl: pastohal, DANS LI: VAl, D'ARIÊG:

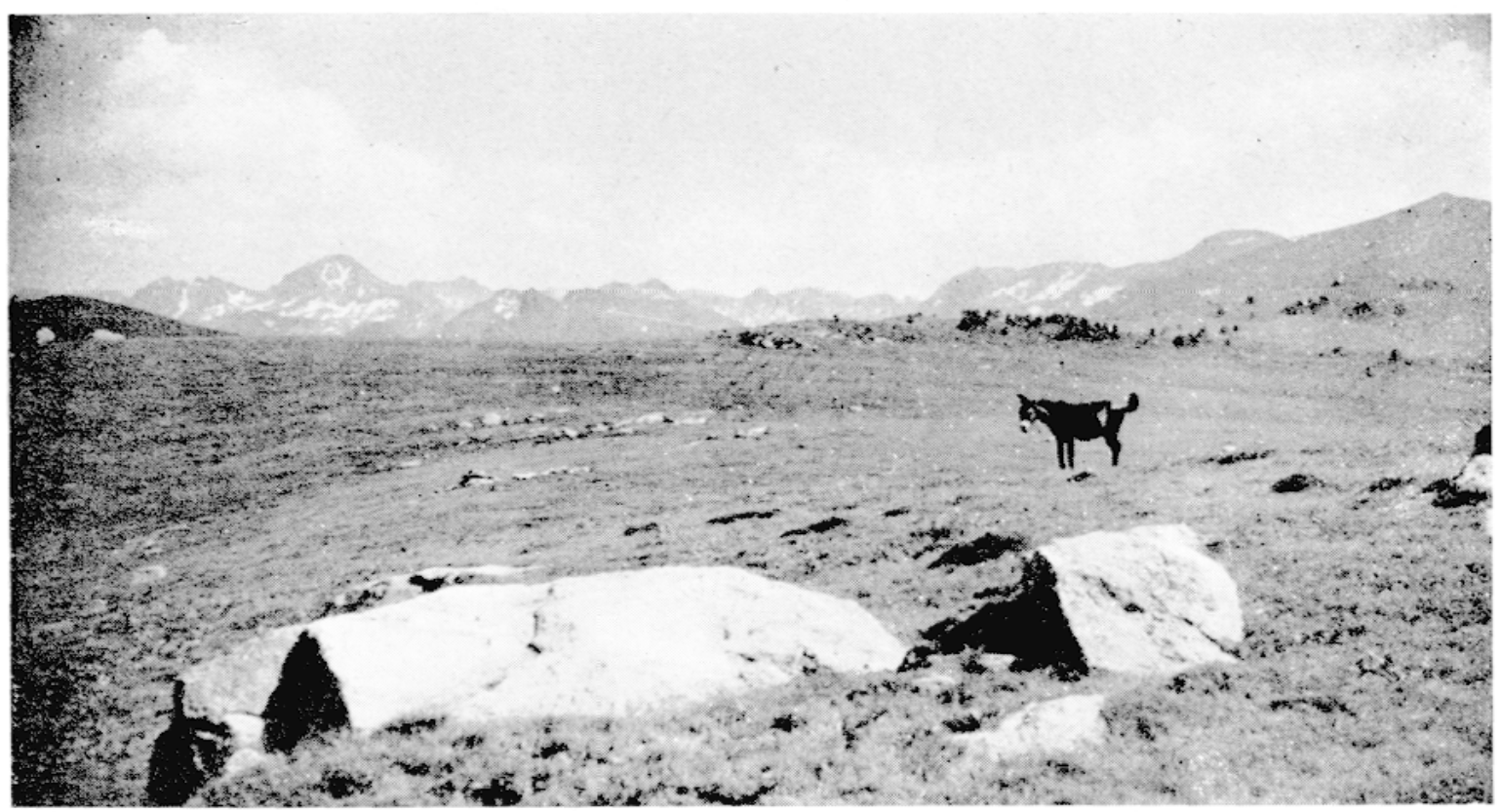

1. Jasse de Beille sur les hautes pelouses du Massif de l'Aston (1 800$2000 \mathrm{~m}$ ). Chaine frontiere de l'An lorre avee, à gauche. le lic de hulle (2 $788 \mathrm{~m}$ ).

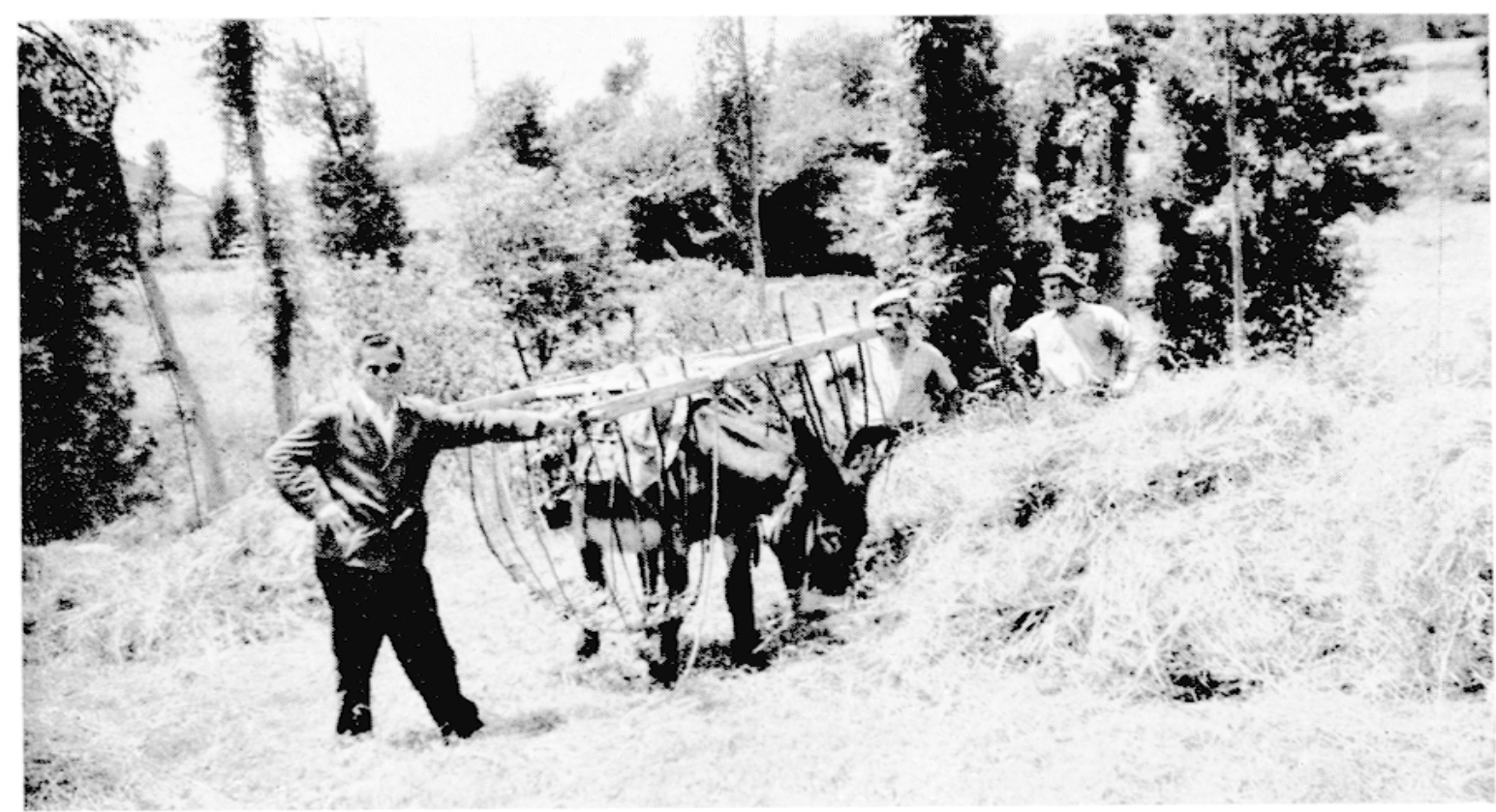

(clichis (i. Bertrand.)

B. Fenaison sur la soulane du Tabe (commune d'Appy). I.e foin coups a la faux est transporte a dos dane dans une balangoire. 


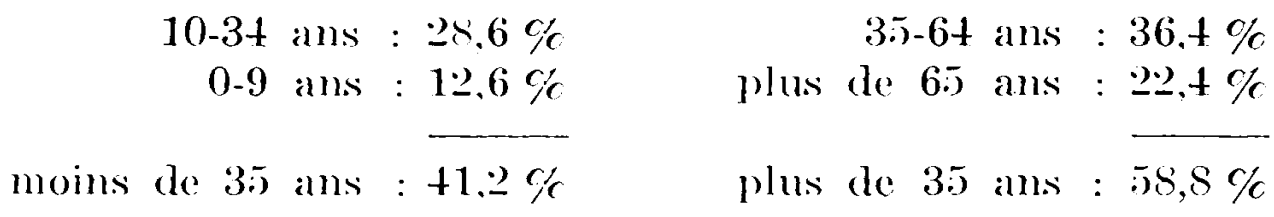

Cependant, le taux de natalité demente tres bas $(20,6 \%)$ ef le remplacement de la population n'est pas assuré.

De plus en plus, il semble que le maintion de la vie pastorale soit

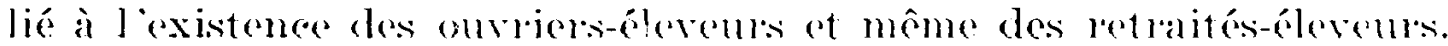
Son principal mérite est dempêeher la prolétarisation d ime grande partie de la population montagnarde et de contribuer de facon sensible à lamélionation du nivean de vie. cela présente plusiems dangers. Tout dabored, es gonles de vie mixtes demement fiagiles ot transitoires. Enfin, les elereurs constituent un ensemble toop hétéroclite pour assurer le progrès on même le maintien de léeonom pastorale. Labsenee de syondieats et de conperatives se fiat lourdement sentir. ('e sont les mumicipalités, dù les éleveurs ne sont sourent qu iune minerité, qui assurent tant hien que mal looranisation de lestivage. La vie pastorale nen demenre pas moins une réalité présente. 